\title{
EDITORIAL
}

\section{Lung volume reduction surgery for emphysema: answers are beginning to accumulate}

\author{
A.B. Thompson
}

End-stage emphysema is a devastating disease, associated with marked morbidity and mortality. The onset of dyspnoea is insidious and unrelenting. By the time medical attention is sought the disease has often progressed to severe, fixed airflow limitation. Medically, the therapeutic options for emphysema have limited efficacy because none can address the anatomical abnormalities which cause the pathophysiological changes seen in emphysema. Lung transplantation is an option for patients with severe emphysema, but advanced age and co-morbid medical conditions often preclude patients with emphysema from being candidates for lung transplantation. Lung volume reductive surgery (LVRS) has emerged as a viable alternative for these patients. Recent publications have begun to address important clinical and mechanistic questions still unanswered about this new procedure.

Clearly LVRS improves dyspnoea, oxygenation, and exercise tolerance for the majority of carefully selected patients. Several centres have confirmed the improvements in respiratory function, exercise tolerance and symptoms first reported by COOPER [1] and others [25]. Surgical stapling of lung tissue appears to be more efficacious and safer than laser ablation [3, 4]. Bilateral resection has yielded larger improvements than unilateral resection with gains in forced expiratory volume in one second (FEV1) ranging $27-34 \%$ for unilateral procedures and $82-96 \%$ for bilateral procedures [1-5]. However, the impact of LVRS on survival and longterm outcome remains to be answered. Moreover, the physiological basis for the clinical improvements has not been elucidated.

Several mechanisms have been postulated for the improvements seen after LVRS. The hyperinflation which complicates emphysema is due to a destruction of lung parenchyma with resulting loss of elastic recoil and tethering open of small airways. Thus, there is both loss of expiratory driving force and increased resistance to airflow during exhalation. The distribution of emphysema is typically quite heterogeneous with the result that more affected areas remain inflated throughout the respiratory cycle and act like space-filling lesions, displacing more functional lung and exaggerating hyperinflation. It has been postulated that, by resecting areas of lung which are the most severely involved by emphysema, elastic recoil and airway tethering would improve thereby improving both the expiratory driving pressure as

Correspondence: A.B. Thompson, Section of Pulmonary and Critical Care Medicine, Dept of Internal Medicine, University of Nebraska Medical Center, Omaha, USA. well as the resistance to airflow. This in turn would lessen hyperinflation and a significant improvement in the geometry of the chest wall would result as $25-30 \%$ of the lung volume is resected.

ScIurba et al. [6] have provided evidence that elastic recoil is improved by LVRS. A majority ( $80 \%$ ) of their patients demonstrated an increased coefficient of retraction following LVRS. While the increase in elastic recoil did not correlate with the improvements seen in lung volumes or spirometric indices, patients who did not have an improvement in elastic recoil also did not increase their exercise tolerance, as measured by six minute walks.

ScIURBA et al. [6] also addressed right ventricular function. They demonstrated improvement in right ventricular function by two-dimensional echocardiography. While they did not directly determine the mechanism for the improvement, several mechanisms for cardiovascular dysfunction have been described including increased pulmonary vascular resistance, impaired atrial filling, and reduced peripheral venous return during inspiration $[7$, 8].

Interestingly, improvement of airflow obstruction is proving not to be the principle mechanism for the relief of dyspnoea. Improvement in the FEV1 does not correlate well with either lessening of dyspnoea or with improvement in exercise tolerance. This is entirely in keeping with the observation that, in chronic obstructive pulmonary disease (COPD), dyspnoea is better related to respiratory muscle function than to airflow obstruction $[9,10]$. Airtrapping and the resulting shift of tidal respiration to higher lung volumes has a significant effect on functional respiratory muscle strength. Respiratory muscle strength, measured by transdiaphragmatic pressure, has been shown to be impaired in patients with COPD, but only as a function of increased lung volume. When transdiaphragmatic pressures were measured at the same lung volumes in normals and in subjects with COPD, the pressures were actually found to be better in the subjects with COPD [11]. These observations reflect the fact that striated muscles demonstrate progressive weakening as they are shortened. Thus, hyperinflation with depression and shortening of the diaphragm results in lower force generation. Furthermore, the geometry of the insertion of the diaphragms is altered by hyperinflation. Loss of the normal domed shape of the diaphragm results in the loss of the expansion of the lower chest wall during inspiration and, in the most severely affected individuals, inward movement of the lower chest wall.

The study by Teschler et al. [12], in this volume of the Journal, addresses the possibility that the efficacy of 
respiratory muscle function is improved by LVRS. The increases seen in maximal inspiratory pressure, sniff nasal inspiratory pressure, and sniff transdiaphragmatic pressure suggest that respiratory muscle function is improved, presumably by improving mechanical efficiency. The study has several shortcomings, all of which may have led to underestimation of the improvement in respiratory muscle function which could possibly result from LVRS. The first is that the LVRS was performed unilaterally. As mentioned above, unilateral LVRS has been shown to yield smaller gains in respiratory function than bilateral procedures and even more importantly for respiratory muscle function, smaller improvements in lung volumes. Transdiaphragmatic pressures were measured with voluntary respiratory efforts. This method yields results that are reliably lower than if the diaphragm is stimulated to contract by stimulation of the phrenic nerve. Finally, the measurements were made at one month after surgery, a time when patients are still recovering from the acute effects of the surgery. In spite of these shortcomings, LVRS was demonstrated to improve respiratory muscle function.

TESCHLER et al. [12] found a decrease in respiratory drive as evidenced by a fall in the mouth occlusion pressure $\left(P_{0.1}\right)$ in their patients following LVRS. $P_{0.1}$ is an easily assessed, noninvasive measure of respiratory drive. Respiratory drive is elevated in patients with COPD and increases as airway obstruction worsens. This is believed to be due to a combination of factors. Early in emphysema, minute ventilation increases to compensate for ventilation/perfusion mismatching. As elastic recoil, airway resistance and hyperinflation worsen, tidal breathing is displaced to higher lung volumes. This results in tidal breathing along higher portions of the pressurevolume curve. To minimize the work of breathing, tidal volumes become smaller and respiratory rate increases. A fall in $P 0.1$ may have resulted, in part, from improved oxygenation, but would also suggest that more efficient patterns of respiration result from LVRS. In addition to the changes in respiratory mechanics, improved respiratory muscle function would improve the efficiency of the coupling of respiratory drive to the generation of negative inspiratory pressure.

These results are consistent with the observation that dyspnoea in patients with COPD is related to respiratory muscle function. By demonstrating that the efficiency of the respiratory muscles was improved by LVRS, the results confirm one possible mechanism for the clinical improvement in patients which results from LVRS.

End-stage emphysema is a common problem in both the Unites States and Europe. In the Unites States, emphysema has a prevalence of 2 million and contributes greatly to the mortality of COPD, the third most common cause of death. Given the high cost of LVRS surgery, the morbidity and mortality associated with the surgery, and the desperation of patients with end-stage emphysema to have something done for their dyspnoea, it is incumbent upon the pulmonary community to carefully define the indications for the surgery and the optimal surgical technique and postoperative care. A full understanding of the physiological consequences of LVRS will provide a rational basis for defining optimal selection criteria. Studies such as the one by TESCHLER et al. [12] will help provide these answers.

\section{References}

1. Cooper JD, Trulock EP, Triantafillou AN, et al. Bilateral pneumonectomy (volume reduction) for chronic obstructive pulmonary disease. J Thorac Cardiovasc Surg 1995; 109: 106-119.

2. Miller JI Jr, Lee RE, Mansour KA. Lung volume reduction surgery: lessons learned. Ann Thorac Surg 1996; 61: 1464-1469.

3. Keenan RJ, Landreneau RJ, Sciurba FC, et al. Unilateral thorascopic surgical approach for diffuse emphysema. $J$ Thorac Cardiovasc Surg 1996; 111: 308-316.

4. McKenna RJ, Brenner M, Gelb AF, et al. A randomized prospective trial of stapled lung reduction versus laser bullectomy for diffuse emphysema. J Thorac Cardiovasc Surg 1996; 111: 317-322.

5. Gaissert HA, Trulock EP, Cooper JD, et al. Comparison of early functional results after volume reduction or lung transplantation for chronic obstructive lung disease. $J$ Thorac Cardiovasc Surg 1996; 111: 296-307.

6. Sciurba F, Rogers RM, Keenan RJ, et al. Improvement in pulmonary function and elastic recoil after lung-reduction surgery for diffuse emphysema. N Engl J Med 1996; 334: 1095-1096.

7. Butler J, Schrijen F, Henriquez A, et al. Cause of the raised wedge pressure on exercise in chronic obstructive pulmonary disease. Am Rev Respir Dis 1988; 138 : 350-354.

8. Nakhjavan FK, Palmer WH, McGregor M. Influence of respiration on venous return in pulmonary emphysema. Circulation 1966; 33: 8-16.

9. Killian K, Jones N. Respiratory muscles and dyspnoea. Clin Chest Med 1988; 9: 237-248.

10. LeBlanc P, Bowie DM, Summers E, et al. Breathlessness and exercise in patients with cardiorespiratory disease. Am Rev Respir Dis 1986; 133: 21-25.

11. Similowski T, Yan S, Gauthier AP, et al. Contractile properties of the human diaphragm during chronic hyperinflation. N Engl J Med 1991; 325: 917-923.

12. Teschler H, Stamatis G, Farhat AA, et al. Effect of surgical lung volume reduction on respiratory muscle function in pulmonary emphysema. Eur Respir $J$ 1996; 9: 1779-1784. 